\title{
MicroRNAs as Novel Biomarkers for the Diagnosis of Alzheimer's Disease and Modern Advancements in the Treatment
}

\author{
Tamil Iniyan Gunasekaran and Takbum $\mathrm{Ohn}^{\dagger}$ \\ Department of Cellular and Molecular Medicine, College of Medicine, Chosun University, Gwangju 501-759, Korea
}

\begin{abstract}
Alzheimer's disease is a common form of dementia occurring among the elderly population and can be identified by symptoms such as cognition impairments, memory loss and neuronal dysfunction. Alzheimer's disease was found to be caused by the deposition of $\beta$-amyloid plaques and neurofibrillary tangles. In addition, mutation in the APP (Amyloid precursor protein), Presenilin 1 (PSEN1) and Presenilin 2 (PSEN2) genes were also found to contribute to Alzheimer's disease. Since the potential conformational diagnosis of Alzheimer's disease requires histopathological tests on brain through autopsy, potential early diagnosis still remains challenging. In recent years, several researches have proposed the use of biomarkers for early diagnosis. In cerebrospinal fluid (CSF), $\beta$-amyloid(1-42), phosphorylated-tau and total tau were suggested to be effective biomarkers for Alzheimer's disease diagnosis. However, a single biomarker might not be sufficient for potential diagnosis of Alzheimer's disease. Thus, the use of RNA interference (RNAi) through microRNAs (miRNAs) has been proposed by several researchers for simultaneous analysis of several biomarkers using microarray technology. These miRNA based biomarkers can be analysed from both blood and CSF, but miRNAs from blood are advantageous over CSF as they are non-invasive and simple for collection. Moreover, the RNAi based therapeutics by siRNA (short interference RNA) or shRNA (short hairpin RNA) have also been proposed to be effective in the treatment of Alzheimer's disease. This review describes the promising application of RNAi technology in therapeutics and as a biomarker for both Alzheimer's disease diagnosis and treatment.
\end{abstract}

Key Words: Alzheimer's disease, Biomarker, miRNA, Diagnosis

\section{INTRODUCTION}

Alzheimer's disease $(\mathrm{AD})$ is a progressive, neurodegenerative disorder characterised by a series of symptoms that includes memory loss, behavioural problems and impairments in the cognition functions, which collectively affects the person's daily activities significantly. Alzheimer's disease is thought to be caused by neuronal dysfunction due to

\footnotetext{
* Received: March 24, 2015 / Revised: March 30, 2015

Accepted: March 30, 2015

${ }^{\dagger}$ Corresponding author: Takbum Ohn. Department of Cellular and Molecular Medicine, College of Medicine, Chosun University, Gwangju 501-759, Korea.

Tel: +82-62-230-6285, Fax: +82-62-233-6337

e-mail: tohn@chosun.ac.kr

(C) The Korean Society for Biomedical Laboratory Sciences. All rights reserved.
}

extracellular $\beta$-amyloid deposition and intracellular tau hyperphosphorylation. It is a common form of dementia occurring mainly in the elderly population. The prevalence rate of Alzheimer's disease was found to be $25 \sim 50 \%$ higher among the people above the age of 85 years (Hong-Qi et al., 2012). In 2011, it was reported that the prevalence of Alzheimer's disease was 33.9 million people all over the world and the prevalence rate is supposed to increase by three-fold in the next forty years (Barnes and Yaffe, 2011). Even though several drug therapies like cholinesterase inhibitors, N-methyl-D aspartate receptor antagonist and some neuroprotective agents are available for $\mathrm{AD}$ treatment, effective disease-modifying therapies are still out of reach (Hong-Qi et al., 2012).

In 1901, a patient named Auguste D, a 51-year-old woman, 
was admitted to the Frankfurt State Asylum with severe progressive impairments in cognition function, delusions, auditory hallucinations, aphasia, erratic behaviour, paranoia and impaired social functioning. After two years, A German physician, Alois Alzheimer joined Munich Medical School to work with Professor Emil Kraepelin, the father of modern psychiatry. Since then, Alzheimer showed special interest towards Auguste D's case. After Auguste D's death on April 8, 1906, Emil Kraepelin encouraged Alzheimer to do research on the patient's brain. As per his request, Alzheimer did an autopsy on her brain using silver staining technique. He found some abnormal changes in her brain, which includes formation of argyrophilic plaques, neurofibrillary tangles and atherosclerotic changes. On November 3, 1906, Alzheimer reported his scientific findings at a conference in Tübingen, Germany (Reger, 2002; Hodges, 2006; Salawu et al., 2011). In 1910, the term "Alzheimer's disease" was coined by Emil Kraepelin to differentiate it from other types of dementia (Zilka and Novak, 2006).

\section{Changes in the Alzheimer's disease affected brain}

After the onset of Alzheimer's disease, the main regions that are affected are the braincortex, entorhinal cortex and hippocampus. Preliminary damages associated with Alzheimer's disease starts from entorhinal cortex in the brain that connects the hippocampus. Later, the disease progression affects the hippocampus, which accounts for memory formation, and followed by the atrophies that further proceeds to the cerebral cortex. As the disease progresses, neuronal loss triggers brain shrinkage and the cerebrospinal fluid (CSF) fills the space caused by it. As the disease reaches its severity, there will be increase in the size of lateral and third ventricles, and this stage eventually end up in long-term memory loss, weight loss and even failure in recognising their own family members (Jackson-Siegal, 2005).

\section{$\beta$-amyloid plaques}

The $\beta$-amyloid $(\mathrm{A} \beta)$ plaques are the resultant of a large amyloid precursor protein (APP), which is an integral membrane glycoprotein. It is usually expressed in the brain and central nervous system, and it undergoes proteolytic $\alpha$ and $\beta$ pathways(Jackson-Siegal, 2005; Dong et al., 2012). The cleavage of APP through $\alpha$ pathway yields nonamyloidogentic product by $\alpha$-secretase and $\gamma$-secretase. But, the $\beta$ pathway cleavage of APP by $\beta$-secretase generates $\beta$-amyloid plaques and the soluble peptide APP $\beta$ ( $\mathrm{SAPP} \beta$ ). This sAPP $\beta$ is released into extracellular space by $\beta$-secretase, leaving behind the 99-amino-acid C-terminal sequence (C99) within the cellular membrane. Then, the $\gamma$-secretase converts C99 into 38-43 amino acids and produces $\beta$ amyloid and APP intracellular C-terminal domain (ACID). However, the cleavage by $\gamma$-secretase generally produces a soluble $A \beta 1-40$, whereas in Alzheimer's disease, the $\gamma$ secretase produces a toxic insoluble A $\beta 1-42$ (JacksonSiegal, 2005; Dong et al., 2012). Mutations in the enzymes that cleave APP causes elevated deposition of $\beta$-amyloid, specifically $A \beta 1-42$, which leads to early-onset familial Alzheimer's disease (EOFAD). The common late-onset Alzheimer's disease (LOAD) is thought to be caused by microglial cells, low level expression of $\beta$-amyloid degrading protease, reduced level of low density lipoprotein receptorrelated protein 1 (LRP1) to clear $\beta$-amyloid from the brain and reduction in the vascular and perivascular drainage in the brain(Mokhtar et al., 2013).

\section{Neurofibrillary tangles}

Neurofibrillary tangles are comprised of highly phosphorylated tau protein, which is associated with microtubules (Brion, 1998). The main proteinaceous component of neurofibrillary tangles is either straight filament (SF) or paired helical filament (PHF) and sometimes, it was also reported that both SF and PHF can be found as hybrid filaments in the $\mathrm{AD}$ patients. Abnormal phosphorylation of tau involves altered conformation, which relocalizes from axonal region to somatodendritic region (Crowther, 1991; Götz et al., 2004). In addition, phosphorylation causes tau to dissociate from microtubules. The accumulation of highly phosphorylated tau proteins in the neurons is also associated with the imbalance between functions of protein kinases and phosphatases acting on tau protein during the early onset of 
Alzheimer's disease (Brion, 1998).

\section{Genes involved in Alzheimer's disease}

\begin{abstract}
Alzheimer's disease exists as early onset form, the familial and the late onset form, the sporadic. Familial Alzheimer's disease (FAD) is a rare Mendelian dominant inheritance that affects patients less than fifty or sixty years of age and is caused due to mutations in APP, Presenilin 1 (PSEN1) and Presenilin 2 (PSEN2) genes. Whereas, several genetic and environmental factors collectively instigate the late onset Alzheimer's disease (LOAD) and it is commonly found among older population. Although there are several genes associated with $\mathrm{LOAD}$, a gene called apolipoprotein E gene (APOE4) found on chromosome 19 is attributed to high risk for LOAD (Whalley, 2001; Barber, 2012). Mutations in APP genes causeabnormal cleavage of $\beta$-secretase which in turn increasesthe production of fibrillogenic $A \beta 42$ over A $\beta 40$ (Götz et al., 2004). Moreover, mutations in PSEN1 and PSEN2 genes are also associated with A $\beta 42$ deposition (Hutton, 1997).Whereas, mutations in APOE gene is associated with novel monofibrils deposition, which precipitates into dense structures (Guerreiro et al., 2012).
\end{abstract}

\section{Biomarkers in disease diagnosis}

Biomarkers are defined as a measurablechanges caused by the molecular alterations that are occurringwithin the biological system including human tissues, cells or fluids. In neurology, the scientists have started employing biomarkers in the diagnosis and treatment related to neuronal disorders. Blood, urine, muscle, nerves, skin, brain and the cerebrospinal fluid also serves effective information about nervous system. There are two main types of biomarkers: biomarkers of exposure and biomarkers of disease. The biomarkers of exposure are used in risk prediction and helps indirectly in the diagnosis, whereas the biomarkers of disease are used directly in the diagnosis, screening and analysing disease progression.

Biomarkers of exposure are further classified into biomarkers of environmental exposures, biomarkers of genetic susceptibility and intermediate biomarkers. Biomarkers of environmental exposures are used when a disease is suspected of being caused due to environmental toxic exposure. Furthermore, the measurement of external dose caused by a specific toxin in the environment along with the internal dose may provide more precise information in the diagnosis. Biomarkers of genetic susceptibility are used to analyse variant alleles in a gene, which is associated to a disease. Currently, biomarkers of genetic susceptibility are increasingly more available for neurological disorders. Intermediate biomarkers are expressed in the pathway of a disease progression. Some of them are related directly or indirectly to a specific disease. Thus, the intermediate biomarkers are strongly correlated to a disease.

Biomarkers of disease are divided into biomarkers of screening or diagnosis, biomarkers variability and biomarkers validity. Biomarkers that are used for screening or diagnosis represent early signs of a disease, which helps in diagnosing a disease at a primitive stage. Cerebrospinal fluid, blood and urine provide essential details for the early diagnosis. Even though biomarkers have several advantages, there is always a concern with measurement errors. Hence, considering the variability factors during the measurement can help in decreasing the misclassification of a disease. Interindividual, intra-individual and group variability are the type of variability occurs during the assessment. Generally, the evaluation of biomarkers reflects its clinical validity. The number of false positives and false negatives should be estimated to analyse the effect of misclassification of disease. Predominantly, the validity of biomarkers is measured by its diagnostic accuracy (Mayeux, 2004; Bossuyt, 2010).

\section{Biomarkers for the diagnosis of Alzheimer's disease}

Although Alzheimer's disease causes several million deaths each year, the potential method of diagnosis is only possible after the histological tests on brain tissues during autopsy (Reitz, 2012; Rosen et al., 2013). However, unambiguous diagnosis of Alzheimer's disease needs both clinical and histopathological post-mortem results. Moreover, the early diagnosis of Alzheimer's disease still remains challenging due to the common symptoms that are similar to 
other neurodegenerative diseases (Humpel, 2011). Since the current method of diagnosis is limited in accuracy, the biomarker based diagnosis of Alzheimer's disease is emerging as a promising method (Fagan and Perrin, 2012). There are three biomarkers to diagnose $\mathrm{AD}$ in $\mathrm{CSF}$ that are internationally validated: $\beta$-amyloid(1-42), phosphorylated-tau and total tau. Nevertheless, the combination of all three of these biomarkers can only provide significant sensitivity to the results.

\section{Inflammatory biomarkers}

The over expression of brain's innate immune responses to Alzheimer's disease triggers inflammatory processes, which in turn stimulates cytokine cellular responses and finally results in neuronal damage. Chronic activation of microglia and astrocytes along with over expression of interleukin-1 (IL-1) and S100 can be found in the brain of AD patients (Mrak and Griffin, 2005).

\section{Novel approach in Alzheimer's disease diagnosis}

It is now evident that a single biomarker isinsufficient for diagnosis of AD. Novel and high-throughput robust approachesare needed for effective diagnosis. Hence, scientists have put forth a modern approach of simultaneous detection of several biomarkers, which proved highly potent enough for AD diagnosis (Humpel, 2011). Gene microarray is one of such high-throughput tool for simultaneous analysis of complex multiple cellular pathway. Genomic level mutations have also been reported in microarrays and helped in studying abnormal expression of genes that were not previously observed during AD diagnosis (Ricciarelli et al., 2004). Due to phylogenetic nature of Alzheimer's disease, large number of genes and their complex pathways need to be examined carefully. With the help of systems biological approach, scientists have studied huge network of co-expressed genes along with their functional modules (Ray et al., 2008). The cDNA microarray is very useful in studying sequence of changes in different gene expression patterns in the brain. This type of study guides us in understanding the deep insights of molecular pathway at every stages of AD (Pasinetti, 2001). In addition, high-density cDNA array is used to characterise mRNA expression levels in thousands of genes simultaneously (Wang et al., 2003).

Apart from other biomarkers, MicroRNA (miRNA) expression levels have been identified as another potential diagnostic biomarker for AD. Since miRNAs circulate in body fluids like plasma, CSF and urine, it is reliable to perform tissue specific profiles (Cheng et al., 2013). MicroRNAs are small $(\sim 22 \mathrm{nt})$ regulatory molecules, which involves in the regulation of specific target mRNA, and it was also found to be playing a vital role in physiological and pathological functions (Mitchell et al., 2008). In 2008, it was reported that miRNAs were found in significant level in the blood and they were also distributed widely in plasma, platelets, nucleated blood cells and erythrocytes. Surprisingly, plasma miRNAs are highly stable under harsh conditions like boiling temperature, long time storage in room temperature, repeated freeze-thaw cycles and at low or high $\mathrm{pH}$. However, the synthetic miRNA were degraded rapidly due to RNase activity in blood plasma. Recent studies revealed that the stability of miRNA is due to packing of miRNAs within the microparticle such as exosomes, microvesicles and apoptotic bodies or with the association of RNAbinding proteins (Argonaute2) or lipoprotein complexes (High Density Lipoprotein) (Creemers et al., 2012). It has been found that more than 1000 different miRNAs are found in human body and their interactions with mRNAs are highly complex. Nearly, one-third of protein encoded genes are found to be regulated by miRNA (Diehl et al., 2012).

Plasma miRNAs are protected against degradation by its packing within protein or lipid vesicles. Based on their size and type of release from the cells, they are divided into exosomes (50 100 nm), membranous vesicles $(0.1 \sim 1 \mu \mathrm{m})$ and apoptotic bodies $(0.5 \sim 2 \mu \mathrm{m})$ (Fig. 1). Scientists reported that circulating miRNA exist as vesicle associated and non-vesicle associated. Approximately $90 \%$ of miRNAs in the circulation are identified as non-membrane-bound, while a small amount of circulating miRNAs are membranebound. Thus, the non-membrane-bound circulating miRNA are associated with Ago2 to form protein complex, which in turn helps it to evadeRNase degradation. HDL particles are much smaller than exosomes and it binds to extra- 


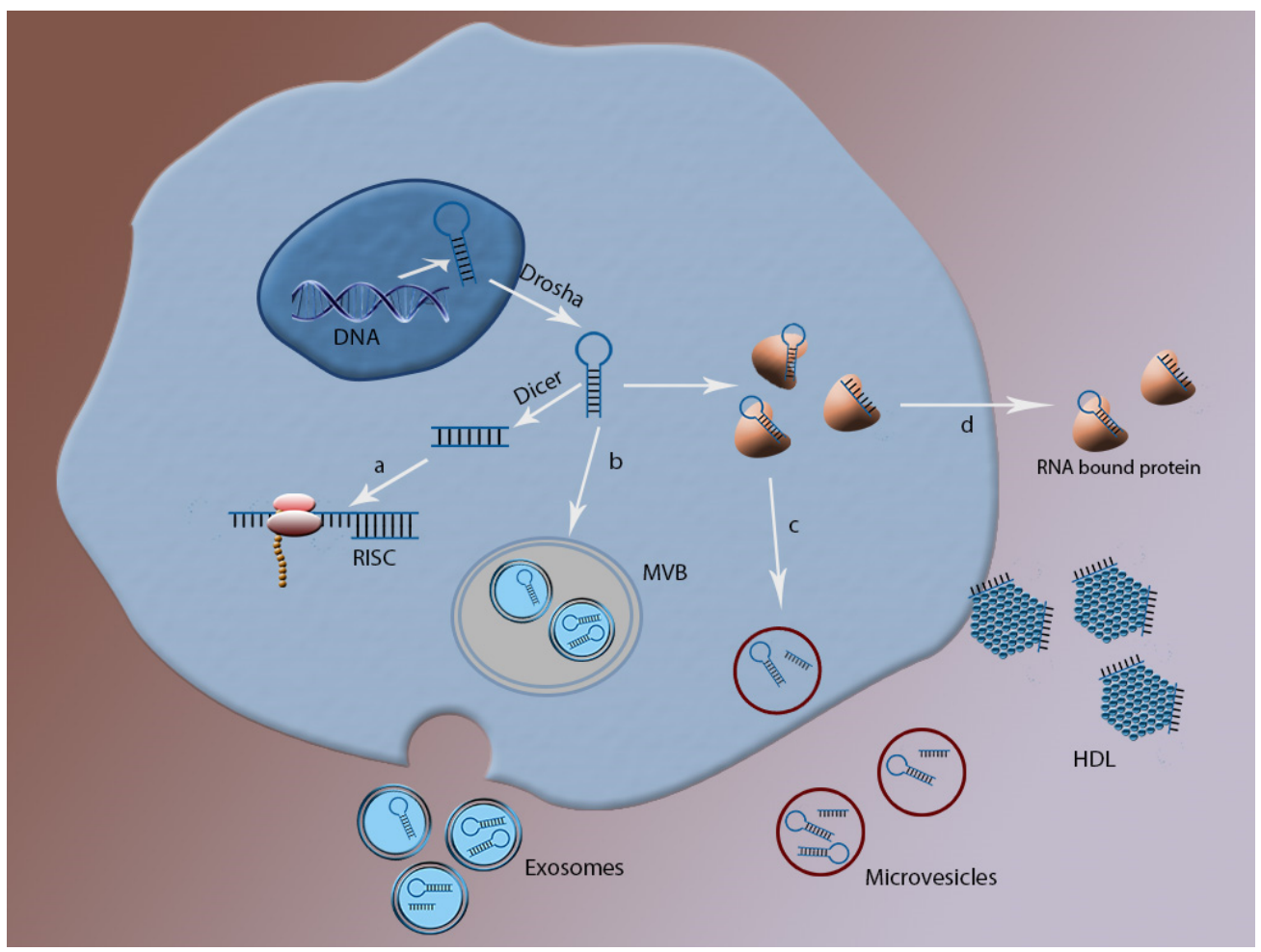

Fig. 1. Pathway of circulating microRNA. The figure shows the miRNAs are transcribed from the DNA within the cellular nucleus. Then, the pre-miRNA are formed by RNAase III enzyme (dorsha) through cleavage and transported into the cytoplasm. Later, the pre-miRNAs are further cleaved into mature miRNA duplexes. (a.) One strand of miRNA duplex silences a specific mRNA by loading into the RNA-induced silencing complex (RISC). (b.) The other miRNA duplex may get degraded or exported out of the cell by packed inside exosomes, which is formed when multivesicular bodies (MVB) fuse with plasma membrane. (c.) Cytoplasmic miRNAs exported by blebbing of plasma membrane and released within microvesicles (d.) miRNAs are released in protein bound form. Usually protein bound miRNAs are bound with RNA binding like with Ago2, HDL and NPM1 (Creemers et al., 2012).

cellular miRNAs by a divalent cation bridge. Moreover, the miRNAs that are associated with HDL were found to be approximately 22 nucleotides in length, which signifies that they are mature miRNAs. However, the function of mature miRNA is still unknown as only pre-miRNA is involved in RNA-induced silencing mechanism (Creemers et al., 2012). Several studies have reported that the circulating miRNA as a potential non-invasive biomarker for a number of human cancers (Tricoli and Jacobson, 2007), cardiovascular diseases(Creemers et al., 2012), multiple sclerosis (Gandhi et al., 2013), diabetes mellitus (Guay and Regazzi, 2013) and several neurodegenerative diseases (Du and Pertsemlidis, 2011).

\section{MicroRNAs in Alzheimer's disease}

In $\mathrm{AD}$, miRNAs are differentially expressed and plays a crucial role in the regulation of pathogenesis. Modern advancements enable high-throughput evaluation of miRNA functions in genome-wide format (Tan et al., 2014). Since the miRNAs are playing a vital role in brain development and neuronal functions, the deregulated brain miRNAs along with CSF miRNAs can be ideally used as biomarkers for AD diagnosis (Cogswell et al., 2008). Nevertheless, circulating miRNAs from blood have good advantage over the $\mathrm{CSF}$, as the circulating miRNAs are non-invasive and does not require any trained physician for sample collection unlike CSF. Circulating miRNAs that are collected from 
blood plasma are used for high-throughput gene expression profiling and can be validated with qRT-PCR (Kumar et al., 2013). Several miRNAs in brain tissues of AD patients also serves as potential biomarkers. Furthermore, researchers are involved in developing advanced techniques to improve the sensitivity and specificity of the body fluid biomarkers (Zilka and Novak, 2006).

\section{MicroRNAs in therapeutics of Alzheimer's disease}

MicroRNAs can be used in therapeutics by constructing a synthetic RNA that reflects the endogenous specific miRNA functions. Artificial miRNAs should be constructed with high stability to counter RNase activity and enable cellular uptake within the host. The construct will have a 'guide strand', which is identical to a specific miRNA of interest and another is a 'passenger strand' that will be linked to a molecule like cholesterol for enabling cellular uptake. Even though the synthetic miRNA functions as endogenous miRNA, the uptake of synthetic miRNA by tissues will results in potential unspecific targeting effects. To avoid side-effects, the specificity of synthetic miRNA should be taken into account. However, adeno-associated viral vectors are now widely used to deliver miRNA, which will allow miRNAs to continuously get expressed. Additionally, several types of adeno-associated virus (AAV) serotypes are now available. Some AAV serotypes will have tissue specific promoters and some carry cellular receptors to increase specificity of the miRNAs (van Rooij et al., 2012). In Alzheimer's disease, RNA interference (RNAi) based therapeutics have been successfully demonstrated by allelespecific gene silencing mechanism through short hairpin RNA (shRNA) or siRNA to specifically suppress mutated APP. Intravenous injection of rabies viral-glycoproteintargeted by exosome mediated delivery of short interference RNA (siRNA) to the neurons of mouse brain resulted in significant reduction of BACE1 ( $\beta$-secretase 1$)$ related mRNAs and proteins (Singer et al., 2005; Shun et al., 2013). Since AD is genetically heterogeneous, it needs to be treated with multiple siRNAs and the formulation of different RNAi components to deliver it accurately to the target sites is a challenging approach (Shun et al., 2013).

\section{CONCLUSION}

$\mathrm{AD}$ is a complex neurodegenerative disease and requires a novel therapeutic strategy to counter it (Lukiw, 2012). Despite several researches for potential biomarkers in blood, CSF, plasma and serum, the application of reliable and sensitive biomarker for $\mathrm{AD}$ diagnosis was not successful. With the help of high-throughput sequencing of miRNA and validation, it is now possible for a cost-effective and non-invasive type of $\mathrm{AD}$ diagnosis. In addition, the miRNA biomarkers that are associated with $\mathrm{AD}$ diagnosis also have potential for the diagnosis of other neurodegenerative diseases like Parkinson's disease, Huntington's disease and Prion disease (Cheng et al., 2013). Circulating blood biomarkers have received substantial attraction due to its positive impact on patient's comfort, which helps in early diagnosis, continuous monitoring and treatment at the primitive stage of AD (Kumar et al., 2013). Since RNAi controls the regulation of gene silencing mechanism, targeting these RNAi mechanism is a novel therapeutic strategy to counter several neurodegenerative diseases (Orlacchio et al., 2007). RNA interference using siRNA has become a promising tool for a gene or allele specific suppression of disease related mRNAs (Koutsilieri et al., 2007). To avoid nonspecific targeting actions by synthetic miRNAs, the specific siRNA of interest needs to be delivered through suitable viral vectors for continuous expression (van Rooij et al., 2012). Potential use of siRNA as a novel therapeutic agent against several neurodegenerative diseases including AD have already been demonstrated in several researches. Moreover, RNAi technology will likely play a major leading role in both diagnosis and therapeutics of $\mathrm{AD}$ for the foreseeable future.

\section{Acknowledgement}

This study was supported by a research fund from Chosun University, 2013.

\section{REFERENCES}

Barber RC. The Genetics of Alzheimer's Disease. Scientifica. 
2012. 2012: 14 .

Barnes DE, Yaffe K. The projected effect of risk factor reduction on Alzheimer's disease prevalence. Lancet Neurol. 2011. 10: 819-828.

Bossuyt P. Clinical validity: defining biomarker performance. Scand J Clin Lab Invest Suppl. 2010. 242: 46-52.

Brion J. Neurofibrillary tangles and Alzheimer's disease. Eur Neurol. 1998. 40: 130-140.

Cheng L, Quek C, Sun X, Bellingham SA, Hill AF. Deepsequencing of microRNA associated with Alzheimer's disease in biological fluids: From biomarker discovery to diagnostic practice. Fron Genet. 2013. 4: 150.

Cogswell JP, Ward J, Taylor IA, Waters M, Shi Y, Cannon B, Kelnar K, Kemppainen J, Brown D, Chen C, Prinjha RK, Richardson JC, Saunders AM, Roses AD, Richards CA. Identification of miRNA changes in Alzheimer's disease brain and CSF yields putative biomarkers and insights into disease pathways. J Alzheimers Dis. 2008. 14: 27-41.

Creemers EE, Tijsen AJ, Pinto YM. Circulating microRNAs: Novel biomarkers and extracellular communicators in cardiovascular disease? Circ Res. 2012. 110: 483-495.

Crowther RA. Straight and paired helical filaments in Alzheimer disease have a common structural unit. Proc Natl Acad Sci USA. 1991. 88: 2288-2292.

Diehl P, Fricke A, Sander L, Stamm J, Bassler N, Htun N, Ziemann M, Helbing T, El-Osta A, Jowett JBM, Peter K. Microparticles: major transport vehicles for distinct microRNAs in circulation. Cardiovasc Res. 2012. 93: 633-644.

Dong S, Duan Y, Hu Y, Zhao Z. Advances in the pathogenesis of Alzheimer's disease: a re-evaluation of amyloid cascade hypothesis. Transl Neurodegener. 2012. 1: 18.

Du L, Pertsemlidis A. Cancer and neurodegenerative disorders: pathogenic convergence through microRNA regulation. J Mol Cell Biol. 2011. 3: 176-180.

Fagan AM, Perrin RJ. Upcoming candidate cerebrospinal fluid biomarkers of Alzheimer's disease. Biomark Med. 2012. 6: 455-476.

Götz J, Schild A, Hoerndli F, Pennanen L. Amyloid-induced neurofibrillary tangle formation in Alzheimer's disease: insight from transgenic mouse and tissue-culture models. Int J Dev Neurosci. 2004. 22: 453-465.

Gandhi R, Healy B, Gholipour T, Egorova S, Musallam A, Hussain MS, Nejad P, Patel B, Hei H, Khoury S, Quintana F, Kivisakk P, Chitnis T, Weiner HL. Circulating MicroRNAs as biomarkers for disease staging in multiple sclerosis. Ann
Neurol. 2013. 73: 729-740.

Guay C, Regazzi R. Circulating microRNAs as novel biomarkers for diabetes mellitus. Nat Rev Endocrinol. 2013. 9: 513-521.

Guerreiro RJ, Gustafson DR, Hardy J. The genetic architecture of Alzheimer's disease: beyond APP, PSENs and APOE. Neurobiol Aging. 2012. 33: 437-456.

Hodges JR. Alzheimer's centennial legacy: origins, landmarks and the current status of knowledge concerning cognitive aspects. Brain. 2006. 129: 2811-2822.

Hong-Qi Y, Zhi-Kun S, Sheng-Di C. Current advances in the treatment of Alzheimer's disease: focused on considerations targeting Abeta and tau. Transl Neurodegener. 2012. 1: 21.

Humpel C. Identifying and validating biomarkers for Alzheimer's disease. Trends Biotechnol. 2011. 29: 26-32.

Hutton M. The presenilins and Alzheimer's disease. Hum Mol Genet. 1997. 6: 1639-1646.

Jackson-Siegal J. Our current understanding of the pathophysiology of Alzheimer's disease advanced studies in pharmacy. Adv Stud Pharm. 2005. 2: 126-135.

Koutsilieri E, Rethwilm A, Scheller C. The therapeutic potential of siRNA in gene therapy of neurodegenerative disorders. J Neural Transm Suppl. 2007. 72: 43-49.

Kumar P, Dezso Z, MacKenzie C, Oestreicher J, Agoulnik S, Byrne M, Bernier F, Yanagimachi M, Aoshima K, Oda Y. Circulating miRNA biomarkers for Alzheimer's disease. PLoS One. 2013. 8: e69807.

Lukiw W. NF-kappaB-regulated, proinflammatory miRNAs in Alzheimer's disease. Alzheimers Res Ther. 2012. 4: 47.

Mayeux R. Biomarkers: potential uses and limitations. Neurotherapeutics. 2004. 1: 182-188.

Mitchell PS, Parkin RK, Kroh EM, Fritz BR, Wyman SK, Pogosova-Agadjanyan EL, Peterson A, Noteboom J, O'Briant KC, Allen A, Lin DW, Urban N, Drescher CW, Knudsen BS, Stirewalt DL, Gentleman R, Vessella RL, Nelson PS, Martin DB, Tewari M. Circulating microRNAs as stable blood-based markers for cancer detection. Proc Natl Acad Sci. 2008. 105: 10513-10518.

Mokhtar SH, Bakhuraysah MM, Cram DS, Petratos S. The beta-amyloid protein of Alzheimer's disease: Communication breakdown by modifying the neuronal cytoskeleton. Intern J Alzheimers Dis. 2013. 2013: 15.

Mrak RE, Griffin WST. Potential inflammatory biomarkers in Alzheimer's disease. J Alzheimers Dis. 2005. 8: 369-375.

Orlacchio A, Bernardi G, Orlacchio A, Martino S. RNA interference as a tool for Alzheimer's disease therapy. Mini Rev Med 
Chem. 2007. 7: 1166-1176.

Pasinetti GM. Use of cDNA microarray in the search for molecular markers involved in the onset of Alzheimer's disease dementia. J Neurosci Res. 2001. 65: 471-476.

Ray M, Ruan J, Zhang W. Variations in the transcriptome of Alzheimer's disease reveal molecular networks involved in cardiovascular diseases. Genome Biol. 2008. 9: R148.

Reger B. Alzheimer's Disease: A brief history and avenues for current research. J Young Invest. 2002. 6.

Reitz C. Alzheimer's disease and the amyloid cascade hypothesis: A critical review. Intern J Alzheimers Dis. 2012. 2012: 11.

Ricciarelli R, d'Abramo C, Massone S, Marinari UM, Pronzato MA, Tabaton M. Microarray analysis in Alzheimer's disease and normal aging. IUBMB Life. 2004. 56: 349-354.

Rosen C, Hansson O, Blennow K, Zetterberg H. Fluid biomarkers in Alzheimer's disease - current concepts. Mol Neurodegener. 2013. 8: 20

Salawu F, Umar J, Olokoba A. Alzheimer's disease: A review of recent developments. Ann Afr Med. 2011. 10: 73-79.

Shun C, Xuemei G, Yinghui C, Nan L, Zhenguo L, Weien Y. Advances with RNA interference in Alzheimer's disease research. Drug Des Devel Ther. 2013. 7: 117-125.

Singer O, Marr RA, Rockenstein E, Crews L, Coufal NG, Gage FH, Verma IM, Masliah E. Targeting BACE1 with siRNAs ameliorates Alzheimer disease neuropathology in a transgenic model. Nat Neurosci. 2005. 8: 1343-1349.

Tan L, Yu JT, Tan MS, Liu QY, Wang HF, Zhang W, Jiang T, Tan L. Genome-wide serum microRNA expression profiling identifies serum biomarkers for Alzheimer's disease. J Alzheimers Dis. 2014. 40: 1017-1027.

Tricoli JV, Jacobson JW. MicroRNA: potential for cancer detection, diagnosis, and prognosis. Cancer Res. 2007. 67: 4553-4555.

van Rooij E, Purcell AL, Levin AA. Developing microRNA therapeutics. Circ Res. 2012. 110: 496-507.

Wang G, Zhang Y, Chen B, Cheng J. Preliminary studies on Alzheimer's disease using cDNA microarrays. Mech Ageing Dev. 2003. 124: 115-124.

Whalley LJ. Early-onset Alzheimer's disease in Scotland: environmental and familial factors. Brit J Psychiat. 2001. 178: s53 -s59.

Zilka N, Novak M. The tangled story of Alois Alzheimer. Bratisl Lek Listy. 2006. 107: 343-345. 